\title{
Gene frequencies in the blood group systems of the Cuban Charolais
}

\author{
Miriam RIBAS \\ Instituto de Ciencia Animal, Apartado 24, \\ San José de Las Lajas, La Habana, Cuba
}

\begin{abstract}
Summary
A total of 223 dam-son pairs of the Cuban Charolais breed were used to establish gene frequencies in the following eleven blood group systems : systems $\mathrm{A}, \mathrm{B}, \mathrm{C}, \mathrm{F}, \mathrm{J}, \mathrm{L}, \mathbf{M}$, $\mathbf{S}, \mathbf{Z}, \mathbf{R}^{\prime}, \mathbf{T}^{\prime}$. In the $\mathrm{F}$ system the population was found to be in Hardy-Weinberg equilibrium. Fifty eight alleles were found in the B system and forty three alleles in the $C$ system. The ten most frequent alleles in the Cuban Charolais are not encountered in the Holstein, Santa Gertrudis, Criollo or Zebu breeds found in Cuba. The allele $\mathrm{SU}^{\prime \prime}\left(\mathrm{U}^{\prime}\right.$ without $\mathrm{U}_{2}^{\prime}$ ) not found in European breeds was present in the S system. It was concluded that the Cuban Charolais showed a high variability and was decisively different from other breeds of cattle found in Cuba. The presence of the $\mathrm{SU}^{\prime \prime}$ allele would seem to indicate that Bos indicus genes were present in Cuban Charolais Cattle.
\end{abstract}

\section{I. - Introduction}

Ribas \& MrTat (1975) reported that the Cuban Charolais breed, which was well adapted to the climate and was found mainly in the «Manuel Fajardo » Genetic Centre in Jiguani, Granma Province, had its origins in animals imported from France at the beginning of the century. As with the French Charolais the growth and carcass traits (Willis \& Preston, 1967 ; Willis, 1969 ; Willis, Preston \& Menchaca, 1974 ; Willis \& Preston, 1970) of the Cuban Charolais were good, however, they did not have the calving difficulties associated with the French breed. This indicated certain differences in the genetic constitution of the breed which might be analysed with the aid of blood groups.

The objective of the present work was to establish the gene frequencies of eleven blood group systems in Cuban Charolais Cattle.

\section{II. - Material and methods}

Samples were taken from the herd standing at the "Manuel Fajardo " Genetic Centre in Jiguani. Approximately 17 p. 100 of the herd (223 dam-son pairs) was randomly sampled in order to determine the gene frequencies of the different blood group systems. 
Approximately $10 \mathrm{ml}$ of blood was taken from the jugular vein of each animal. Samples were collected with the following anticoagulant solution : sodium citrate : $20 \mathrm{~g}$; sodium chloride : $5 \mathrm{~g}$; sodium cyanide : $0,4 \mathrm{~g}$; distilled water to $1000 \mathrm{ml}$.

A total of 75 reagents detecting factors of 11 blood group systems and produced in the Laboratory of Biochemical Genetics Department of animal genetics I.N.R.A. at Jouy-en-Josas (France) were used. A system : A, $\mathbf{Z}^{\prime}$; B system : $B_{1}, B_{2}, G_{1}, G_{2}, G_{3}, I_{1}, I_{2}, K, O_{1}, O_{3}, O_{x}, P_{1}, P_{2}, Q, T, Y_{2}, A^{\prime}, B^{\prime}, D^{\prime}$, $\mathrm{E}_{1}^{\prime}, \mathrm{E}_{2}^{\prime}, \mathrm{E}_{3}^{\prime}, \mathrm{E}_{4}^{\prime}, \mathrm{G}^{\prime}, \mathrm{I}_{1}^{\prime}, \mathrm{I}_{2}^{\prime}, \mathrm{J}_{1}^{\prime}, \mathrm{J}_{2}^{\prime}, \mathrm{K}^{\prime}, \mathrm{O}^{\prime}, \mathrm{P}_{1}^{\prime}, \mathrm{P}_{2}^{\prime}, \mathrm{Q}^{\prime}, \mathrm{Y}^{\prime}, \mathrm{A}^{\prime \prime}, \mathrm{B}^{\prime \prime}, \mathrm{G}^{\prime \prime}, \mathrm{I}^{\prime \prime}, \mathrm{F} 16$, F18, F20 ; C system : $C_{1}, C_{2}, E, R_{1}, R_{2}, W, X_{1}, X_{2}, C^{\prime}, L^{\prime}, C^{\prime \prime}, F 1, F 6, F 10, F 15$; F system : F, V ; J system : J ; L system : L ; M system : $\mathbf{M}^{\prime}, \mathbf{M}_{1} ; \mathrm{S}$ system : $\mathrm{S}, \mathrm{S}^{\prime \prime}$, $\mathrm{U}, \mathbf{H}^{\prime \prime}, \mathrm{U}^{\prime}, \mathrm{U}^{\prime}{ }_{2}, \mathrm{U}^{\prime \prime}, \mathrm{H}^{\prime} ; \mathrm{Z}$ system : $\mathrm{Z} ; \mathrm{R}^{\prime}$ system : $\mathrm{R}^{\prime} ; \mathrm{T}^{\prime}$ system : $\mathrm{T}^{\prime}$.

The reagents $F 1$ to $F 20$ were experimental reagents produced by the Jouy-enJosas Laboratory.

In the $\mathrm{F}$ system the gene frequencies were estimated by directly counting the three genotypes. The square root method (CoTTERMAN, 1954) was used for the simple systems, while in the complex systems the iterative method was applied (CePpelini et al., 1956 ; NeIMANN-Sorensen, 1956).

\section{III. - Results and discussion}

\section{A. - Test for genetic equilibrium}

To test whether the population appeared to be in genetic equilibrium the observed numbers of the three genotypes of the $F$ system were compared with the expected numbers (table 1). The population was found to be in equilibrium.

\section{TABLE 1}

Genetic equilibrium test in the $F$ system

Test de l'équilibre génétique au système $F$

\begin{tabular}{l|l|l|l|c}
\hline Genotype & F/F & F/V & V/V & Total \\
\hline $\begin{array}{l}\text { Observed } \ldots \ldots \ldots \ldots \ldots \ldots \ldots \ldots \ldots \\
\text { Expected } \ldots \ldots \ldots \ldots \ldots \ldots \ldots \ldots\end{array}$ & 124 & 82 & 17 & 223 \\
\hline
\end{tabular}

$\chi^{2}=0.4321 \mathrm{df}$.

\section{B. - $F, L, J, M, Z, R^{\prime}$ and $T^{\prime}$ systems}

Because anti- $S^{\prime}$ was not available in the $R^{\prime}$ system, and because factor $M_{1}$ was not found, all the above systems, except the $F$ system, behave like one factor, two allele systems. It should be noted that the frequency of the $\mathbf{M}$ factor was extremely low (0.004 : only two animals were found to be positive for this factor).

The gene frequencies obtained in the Cuban Charolais appear in table 2. 
The results agreed with those previously obtained by RoNDA et al. (1971) with the exception of the $Z$ allele which had an increased frequency. This increase may be as much due to the sampling effect as to any possible selection effect caused by greater use of certain bulls (RENDEL, 1963).

\section{TABLE 2}

Gene frequencies in the biallelic systems

Fréquences géniques dans les systèmes bialléliques

\begin{tabular}{|c|c|c|c|c|}
\hline & System & Allele & Frequency & $\mathrm{SE} \pm$ \\
\hline F & & $\mathbf{F}$ & 0.739 & 0.021 \\
\hline $\mathbf{L}$ & $\ldots \ldots \ldots$ & $\mathbf{L}$ & 0.239 & 0.022 \\
\hline $\mathbf{J}$ & $\ldots$ & $\mathbf{J}$ & 0.347 & 0.025 \\
\hline $\mathbf{M}$ & $\ldots \ldots \ldots \ldots \ldots$ & $\mathbf{M}^{\prime}$ & 0.004 & 0.003 \\
\hline $\mathbf{Z}$ & $\ldots \ldots \ldots \ldots \ldots$ & $\mathbf{Z}$ & 0.741 & 0.032 \\
\hline $\mathbf{R}^{\prime}$ & $\ldots \ldots \ldots \ldots \ldots$ & $\mathbf{R}^{\prime}$ & 0.044 & 0.031 \\
\hline$T^{\prime}$ & $\ldots \ldots \ldots \ldots \ldots$ & $\mathrm{T}^{\prime}$ & 0.097 & 0.014 \\
\hline
\end{tabular}

$$
\text { C. - A system }
$$

Although only two reagents were used in the A system, thereby differentiating three phenogroups $\mathrm{A}, \mathrm{AZ}$ and a, this was treated as a complex system, using the iterative method for gene frequency calculations. Results are shown in table 3. They are very similar to those reported by RONDA et al. (1971) in the same breed.

\section{TABLE 3}

Gene frequencies in the $A$ system

Fréquences géniques dans le système $A$

\begin{tabular}{|c|c|}
\hline Phenogroups & Frequencies \\
\hline 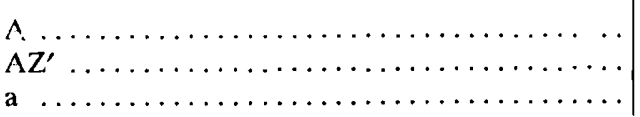 & $\begin{array}{l}0.677 \\
0.225 \\
0.093\end{array}$ \\
\hline
\end{tabular}

D. - B system

Fifty eight phenogroups of the B system were observed. They are presented in table 4 . It should be noted that 1 p. 100 of the alleles present in the breed were not identified due to their low frequencies.

Results presented in this paper differ somewhat from those reported by RiBAs \& Mitat (1975) and KONDA et al. (1971). This may be due to different specificities of the reagents used. 


\section{TABLE 4}

Frequencies of the B system phenogroups in the Cuban Charolais Fréquence des phénogroupes du système $B$ dans la race Charolaise cubaine

\begin{tabular}{|c|c|c|c|}
\hline Phenogroup & Frequency & Phenogroup & Frequency \\
\hline $\mathrm{G}_{3} \mathrm{O}_{1} \mathrm{TE}_{3}^{\prime} \mathrm{I}^{\prime \prime}$ & 0.0798 & $\mathbf{Y Y}^{\prime} \ldots \ldots \ldots \ldots$ & 0.0084 \\
\hline $\mathrm{B}_{2} \mathbf{O}_{1} \mathbf{Y D}^{\prime} \ldots \ldots \ldots \ldots$ & 0.0749 & $\mathrm{YQ}^{\prime} \mathrm{Y}^{\prime} \mathrm{I}^{\prime \prime} \ldots \ldots \ldots$ & 0.0071 \\
\hline $\mathrm{BQG}^{\prime} \mathbf{O}^{\prime} \mathbf{P}^{\prime} \mathbf{B}^{\prime \prime} \ldots \ldots \ldots$ & 0.0662 & $\mathbf{Y I}^{\prime} \mathbf{Q}^{\prime} \mathbf{Y}^{\prime} \quad \ldots \ldots \ldots \ldots \ldots \ldots$ & 0.0067 \\
\hline b $\ldots \ldots \ldots \ldots$ & 0.0662 & $\mathbf{I}_{2} \mathrm{E}_{2}^{\prime} \mathbf{I}^{\prime \prime} \quad \ldots \ldots \ldots \ldots \ldots \ldots$ & 0.0065 \\
\hline$P_{1} E_{4}^{\prime} I^{\prime} I^{\prime \prime} \ldots \ldots$ & 0.0621 & $\mathbf{I}_{3}\left(\mathrm{O}_{1}\right) \mathrm{QE}_{2}^{\prime} \mathrm{I}^{\prime} \mathbf{I}^{\prime \prime}$ & 0.0064 \\
\hline $\mathbf{Y I}^{\prime \prime} \ldots$ & 0.0506 & $G_{1} I_{1} O_{2} D^{\prime} I^{\prime} J^{\prime} K^{\prime} A^{\prime \prime} I^{\prime \prime}$ & 0.0064 \\
\hline $\mathrm{B}_{1} \mathrm{O}^{\prime} \mathrm{I}^{\prime \prime}$. & 0.0431 & $\mathrm{G}_{1} \mathrm{I}_{1} \mathrm{O}_{1} \mathrm{D}^{\prime} \mathrm{Y}^{\prime} \mathrm{K}^{\prime} \mathrm{A}^{\prime \prime} \mathrm{I}^{\prime \prime}$ & 0.0064 \\
\hline $\mathrm{O}_{1} \mathrm{E}_{2}^{\prime} \ldots \ldots \ldots \ldots$ & 0.0342 & BGKYE $_{2}^{\prime} A^{\prime \prime} \quad \ldots \ldots \ldots \ldots \ldots$ & 0.0064 \\
\hline$I^{\prime \prime} \ldots \ldots \ldots \ldots \ldots$ & 0.0338 & $\mathrm{BQ}^{\prime} \mathbf{I}^{\prime \prime} \ldots \ldots \ldots \ldots \ldots \ldots$ & 0.0063 \\
\hline BYP'Y' $^{\prime} \ldots \ldots \ldots \ldots$ & 0.0333 & $\mathbf{B I}^{\prime} \mathbf{Q}^{\prime} \mathbf{I}^{\prime \prime} \ldots \ldots \ldots$ & 0.0048 \\
\hline $\mathrm{BG}_{3} \mathrm{O}_{1} \mathrm{TYE}_{3}^{\prime} \mathrm{I}^{\prime \prime} \quad \ldots$ & 0.0292 & $\mathrm{BG}_{2} \mathrm{KO}^{\prime} \mathrm{I}^{\prime \prime} \ldots$ & 0.0043 \\
\hline $\mathrm{G}_{2} \mathrm{O}_{1} \mathrm{E}_{3}^{\prime} \mathrm{I}^{\prime} \mathrm{K}^{\prime} \mathrm{O}^{\prime} \mathrm{I}^{\prime \prime}$ & 0.0282 & $\mathrm{BG}_{2} \mathrm{O}_{1} \mathrm{P}^{\prime} \mathrm{B}^{\prime \prime} \mathrm{I}^{\prime \prime} \ldots$ & 0.0043 \\
\hline$I^{\prime} Q^{\prime} I^{\prime \prime} \ldots \ldots \ldots$ & 0.0263 & $\mathrm{BG}_{3} \mathrm{TE}_{2}^{\prime} \mathrm{O}^{\prime} \mathbf{P}_{1}^{\prime} \ldots \ldots \ldots \ldots \ldots$ & 0.0043 \\
\hline $\mathrm{G}_{1} \mathrm{O}^{\prime} \mathrm{A}^{\prime \prime} \quad \ldots \ldots \ldots$ & 0.0256 & $\mathrm{BPQE}_{3}^{\prime} \mathrm{P}_{1} \mathrm{Q}^{\prime} \mathrm{I}^{\prime \prime} \quad \ldots \ldots \ldots \ldots \ldots$ & 0.0043 \\
\hline $\mathrm{E}_{3}^{\prime} \mathrm{G}^{\prime} \mathrm{G}^{\prime \prime} \mathrm{I}^{\prime \prime} \quad \ldots \ldots$ & 0.0242 & $\mathrm{O}_{1} \mathrm{~J}^{\prime} \mathrm{K}^{\prime} \mathrm{O}^{\prime} \mathbf{P}^{\prime} \ldots \ldots \ldots$ & 0.0043 \\
\hline $\mathrm{O}^{\prime} \mathrm{I}^{\prime \prime} \mathrm{F} 4 \quad \ldots$ & 0.0224 & $\mathrm{QI}^{\prime \prime} \ldots \ldots \ldots \ldots \ldots$ & 0.0043 \\
\hline $\mathbf{Y E}_{2}^{\prime} \ldots \ldots \ldots \ldots \ldots$ & 0.0192 & $T B^{\prime} E_{3}^{\prime} G^{\prime} O^{\prime} G^{\prime \prime}$ & 0.0043 \\
\hline $\mathrm{G}_{3} \mathrm{O}_{1} \mathrm{TYE}_{3}^{\prime} \mathrm{I}^{\prime \prime} \ldots \ldots \ldots$ & 0.0171 & $\mathrm{YE}_{2}^{\prime} \mathrm{Y}^{\prime} \quad \ldots \ldots \ldots \ldots$ & 0.0043 \\
\hline $\mathbf{P}_{1} \mathbf{E}_{4}^{\prime} \mathbf{I}^{\prime \prime} \quad \ldots \ldots \ldots \ldots$ & 0.0166 & $\mathbf{Q}^{\prime} \ldots \ldots \ldots \ldots \ldots$ & 0.0026 \\
\hline BGKA' $^{\prime} \mathrm{O}^{\prime} \mathrm{A}^{\prime \prime} \mathrm{B}^{\prime \prime} \mathrm{I}^{\prime \prime} \mathrm{F} 4$ & 0.0150 & $\mathrm{BG}_{1} \mathrm{KQO}^{\prime} \mathrm{B}^{\prime \prime} \mathrm{A}^{\prime \prime} \mathrm{I}^{\prime \prime} \mathrm{F} 4$ & 0.0021 \\
\hline$A^{\prime} E_{3}^{\prime} G^{\prime} G^{\prime \prime} F 4 \quad \ldots$. & 0.0150 & $\mathrm{~B}_{2} \mathrm{I}_{1} \mathrm{O}_{3} \mathrm{~A}^{\prime} \mathrm{K}^{\prime} \mathrm{Q}^{\prime} \mathrm{F} 4 \quad \ldots$ & 0.0021 \\
\hline $\mathrm{G}_{3} \mathrm{O}_{3} \mathrm{E}_{2:}^{\prime} \mathrm{J}_{2}^{\prime} \mathrm{I}^{\prime \prime} \mathrm{F} 16$ & 0.0130 & $\mathrm{BI}_{1} \mathrm{Q} \ldots \ldots \ldots \ldots$ & 0.0021 \\
\hline$G_{1} Y^{\prime} B^{\prime} D^{\prime} G^{\prime} Q^{\prime} B^{\prime \prime} I^{\prime \prime}$ & 0.0129 & $\mathrm{BTB}^{\prime} \mathrm{E}_{2}^{\prime} \mathrm{O}^{\prime} \mathrm{P}^{\prime} \mathrm{A}^{\prime \prime I} \mathrm{I}^{\prime \prime} \mathrm{F} 4$ & 0.0021 \\
\hline$Q^{\prime} \mathbf{I}^{\prime \prime} \quad \ldots \ldots \ldots \ldots$ & 0.0109 & $\mathrm{G}_{1} \mathbf{A}^{\prime}\left(\mathbf{I}^{\prime}\right) \mathrm{Y}^{\prime} \mathbf{I}^{\prime \prime} \quad \ldots \ldots \ldots \ldots \ldots$ & 0.0021 \\
\hline $\mathrm{G}_{i 2} \mathrm{I}_{2} \mathrm{Y}^{\prime} \mathrm{O}^{\prime} \ldots \ldots \ldots \ldots$ & 0.0108 & $\mathrm{G}_{2} \mathrm{P}_{1} \mathrm{Q}_{1} \mathrm{TE}_{2}^{\prime} \quad \ldots \ldots \ldots \ldots \ldots$ & C.0021 \\
\hline $\mathrm{BO}_{3} \mathrm{YA}^{\prime} \mathrm{E}_{3}^{\prime} \mathrm{G}^{\prime} \mathrm{P}^{\prime} \mathrm{QG}^{\prime \prime} \mathrm{I}^{\prime \prime}$ & 0.0107 & $\mathrm{P}_{1} \mathrm{Q}_{1} \mathrm{~A}^{\prime} \mathrm{E}_{2}^{\prime} \mathrm{G}^{\prime} \mathrm{P}^{\prime} \quad \ldots$ & 0.0021 \\
\hline B. TP $_{2}^{\prime} A^{\prime \prime} I^{\prime \prime} \quad \ldots \ldots \ldots$ & 0.0086 & $\mathrm{P}_{1} \mathrm{~A}^{\prime} \mathrm{E}^{\prime}, \ldots \ldots$ & 0.0021 \\
\hline $\mathrm{BTB}^{\prime} \mathrm{E}_{2}^{\prime} \mathrm{I}^{\prime} \mathrm{O}^{\prime} \mathrm{P}_{1}^{\prime}{ }_{1} \mathrm{~A}^{\prime \prime} \mathrm{I}^{\prime \prime} \mathrm{F} 4$ & 0.0085 & $\mathrm{YD}^{\prime} \mathrm{E}_{3}^{\prime} \mathrm{G}^{\prime} \mathrm{l}^{\prime \prime} \mathrm{F} 18$ & 0.0021 \\
\hline $\mathrm{O}_{1} \mathbf{Q}_{1} \mathbf{Q}^{\prime} \ldots \ldots \ldots \ldots$ & 0.0085 & $\mathrm{YA}^{\prime} \mathrm{E}_{2}^{\prime} \mathrm{G}^{\prime \prime} \mathrm{F} 4 \quad \ldots$. & 0.0021 \\
\hline
\end{tabular}

A comparison of at least 9 of the most frequent phenogroups of this breed with the phenogroups found in five other breeds in Cuba (Hosltein, Charolais, Santa Gertrudis, Criollo and Zebu) which were studied in the blood groups laboratory of the University of Havana (MrTar, 1975) the following was observed (table 5) : 
TABLE 5

Presence of the most frequent alleles of the Cuban Charolais in other Cuban breeds

Présence des principaux allèles du Charolais cubain dans d'autres races cubaines

\begin{tabular}{|c|c|c|c|c|c|}
\hline & $\begin{array}{c}\text { Charolais } \\
\text { (1) }\end{array}$ & $\begin{array}{c}\text { Holstein } \\
\text { (2) }\end{array}$ & $\begin{array}{c}\text { Santa } \\
\text { Gertrudis } \\
(2)\end{array}$ & $\begin{array}{l}\text { Criollo } \\
\text { (2) }\end{array}$ & $\begin{array}{c}\text { Zebu } \\
(2)\end{array}$ \\
\hline 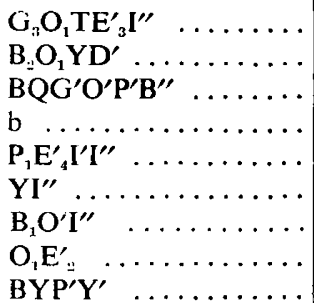 & $\begin{array}{l}\times \\
\times \\
\times \\
\times \\
\times \\
\times \\
\times \\
\times \\
\times\end{array}$ & $\begin{array}{l}\times \\
\times \\
\times\end{array}$ & $\begin{array}{l}\times \\
\times \\
\times\end{array}$ & $\begin{array}{l}\times \\
\times \\
\times\end{array}$ & $x$ \\
\hline
\end{tabular}

(1) Data from the present work.

(2) According to Mitat, 1975.

In Cuba, allele $\mathrm{BG}_{3} \mathrm{O}_{1} \mathrm{TE}_{3}^{\prime} \mathrm{l}^{\prime \prime}$ was only found in the Charolais breed, being the most frequent allele in this breed. The very frequent allele $\mathrm{B}^{\mathrm{BQG}} \mathbf{O}^{\prime} / \mathrm{P}^{\prime} \mathrm{B}^{\prime \prime}$ is characteristic of the Charolais breed in Cuba (Ribas \& Mitat, 1975). On the other hand, the $\mathrm{B}_{3} \mathrm{O}_{1} \mathrm{YD}^{\prime}$ allele was observed in the Holstein, Charolais and Santa Gertrudis.

The allele with the fourth highest frequency in the Charolais breed, the $\mathbf{B}^{\mathbf{b}}$ allele, was found in all other breeds. This was expected because within the $\mathbf{B}^{\mathbf{b}}$ category, alleles may have been included which are not detectable by the reagents currently available.

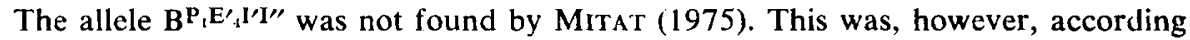
to RiBas \& Mitat (1975), one of the most frequent alleles in the Cuban Charolais breed and in the present sample it had the fifth highest frequency. This allele did not seem to be present in other breeds of cattle in Cuba.

The BYI" allele found in the Cuban Charolais breed was also seen in Hosltein, Santa Gertrudis and Criollo breeds and the $\mathrm{B}^{\mathrm{B}_{1} \mathrm{O}^{\prime} \mathrm{I}^{\prime \prime}}$ allele which MitAT (1975) reported to be only in Zebu cattle was found to be at a high frequency in Cuban Charolais.

The allele $\mathrm{B}^{\mathrm{O}_{1}} \mathrm{E}^{\prime}$, has only been reported in the Cuban Charolais and Criollo breeds (MITAT, 1975). This may indicate the presence of the latter breed in the formation of the Cuban Charolais.

Finally, the allele $\mathrm{B}^{\mathrm{BY}} \mathrm{P}^{\prime} \mathrm{Y}^{\prime}$ has only been found in the Cuban Charolais.

Therefore, the Cuban Charolais appeared to be distinctly different from other breeds found in Cuba. 


\section{E. - C system}

Table 6 presents the forty three phenogroups in the $C$ system. This high number confirmed the genetic variability of the breed, as already observed in the B system.

TABLE 6

Frequencies of the $C$ system phenogroups in the Cuban Charolais breed Fréquences des phénogroupes $d u$ système $C$ dans la race Charolaise cubaine

\begin{tabular}{|c|c|c|c|}
\hline Phenogroup & Frequency & Phenogroup & Frequency \\
\hline $\mathrm{C}_{2} \mathrm{C}^{\prime} \mathrm{F} 6$ & 0.1955 & $\mathrm{C}_{1}$ EWL'F6F10 & 0.0093 \\
\hline $\mathrm{WC}^{\prime \prime} \mathrm{F} 1$ & 0.1077 & $\mathrm{C}_{2} \mathrm{ER}_{1} \mathrm{X}_{1} \mathrm{~F} 6 \mathrm{~F} 15 \quad \ldots$ & 0.0085 \\
\hline$C_{2} R_{2} W^{\prime} C^{\prime} 6 \quad \ldots$ & 0.0989 & $\mathrm{R}_{1} \mathrm{C}^{\prime} \mathrm{C}^{\prime \prime} \mathrm{F} 1,6 \ldots \ldots$ & 0.0085 \\
\hline $\mathrm{R}_{2} \mathrm{WC}^{\prime \prime} \mathrm{F}_{1} \quad \ldots \ldots$ & 0.0796 & $\mathrm{C}_{2} \mathrm{C}^{\prime} \mathrm{L}^{\prime} \mathrm{F} 6 \quad \ldots \ldots \ldots \ldots \ldots$ & 0.0071 \\
\hline $\mathrm{C}_{1}$ EWF6 $\ldots \ldots \ldots \ldots$ & 0.0380 & $\mathrm{C}_{2} \mathrm{EWF} 10 \ldots \ldots$ & 0.0069 \\
\hline $\mathrm{WX}_{2} \mathrm{C}^{\prime \prime} \mathrm{F} 1 \quad \ldots \ldots$ & 0.0363 & $W_{X_{2}} C^{\prime \prime} \ldots \ldots \ldots$ & 0.0068 \\
\hline $\mathrm{R}_{2} \mathrm{C}^{\prime \prime} \mathrm{F} 1,10 \ldots \ldots$ & 0.0305 & $\mathrm{C}_{1} \mathrm{ER}_{2} \mathrm{X}_{1} \mathrm{~L}^{\prime} \mathrm{F} 6 \mathrm{~F} 15$ & 0.0054 \\
\hline$C_{1} E_{2} X_{1} F 6 F 15 \quad \ldots$ & 0.0267 & $\mathrm{C}^{\prime} \mathrm{C}^{\prime \prime} \mathrm{F} 1,6 \ldots \ldots$ & 0.0051 \\
\hline $\mathrm{C}_{2} \mathrm{ER}_{2} \mathrm{X}_{1} \mathrm{~L}^{\prime} \mathrm{F} 6 \mathrm{~F} 15$ & 0.0262 & $\mathrm{C}^{\prime} \mathrm{EF} 6 \ldots \ldots \ldots \ldots$ & 0.0043 \\
\hline $\mathrm{C}_{2} \mathrm{EWX}_{2} \mathrm{~F} 6 \ldots \ldots$ & 0.0261 & $\mathrm{ER}_{1} \mathrm{X}_{2} \mathrm{C}^{\prime \prime} \mathrm{F} 1,6 \quad \ldots$ & 0.0043 \\
\hline $\mathrm{R}_{2} \mathrm{C}^{\prime \prime} \mathrm{F} 6 \quad \ldots \ldots \ldots$ & 0.0261 & $\mathrm{wX}_{2} \quad \ldots \ldots \ldots \ldots \ldots \ldots \ldots$ & 0.0034 \\
\hline$C_{2} R_{1} F 10 \ldots \ldots$ & 0.0205 & $\mathrm{C}_{2} \mathrm{EWX}_{2} \mathrm{C}^{\prime \prime} \mathrm{F} 6 \quad \ldots \ldots \ldots \ldots \ldots$ & 0.0033 \\
\hline $\mathrm{WC}^{\prime \prime} \mathrm{F} 1,10 \ldots$ & 0.0186 & $\mathrm{R}_{\mathbf{1}} \mathrm{F} 10 \ldots \ldots \ldots \ldots \ldots \ldots$ & 0.0029 \\
\hline $\mathrm{C}_{1} \mathrm{EW} \quad \ldots \ldots$ & 0.0180 & EWC ${ }^{\prime \prime} \mathrm{F} 1,6 \ldots \ldots \ldots \ldots \ldots$ & 0.0026 \\
\hline$W^{\prime \prime} \ldots \ldots$ & 0.0155 & $\mathrm{R}_{1} \mathrm{WC}^{\prime \prime} \mathrm{F} 1,10 \ldots \ldots \ldots \ldots$ & 0.0025 \\
\hline c $\ldots \ldots$. & 0.0149 & $\mathrm{C}_{2} \mathrm{EX}_{1} \mathrm{~L}^{\prime} \overline{\mathrm{r}} 6 \mathrm{~F} 15$ & 0.0024 \\
\hline $\mathbf{w} \ldots \ldots \ldots$ & 0.0135 & $\mathrm{C}_{2} \mathrm{EF} 6 \ldots \ldots \ldots \ldots \ldots \ldots$ & 0.0024 \\
\hline $\mathrm{WL}^{\prime} \mathrm{C}^{\prime \prime} \mathrm{F} 1,10$ & 0.0128 & $\mathrm{C}_{1} \mathrm{EX}_{2} \mathrm{~L}^{\prime} \mathrm{F} 6 \ldots \ldots \ldots \ldots \ldots$ & 0.0023 \\
\hline $\mathrm{C}_{2} \mathrm{WC}^{\prime} \quad \ldots \ldots \ldots$ & 0.0124 & $\mathrm{C}_{1} \mathrm{EWX}_{2} \mathrm{C}^{\prime \prime} \mathrm{F} 1 \quad \ldots \ldots \ldots \ldots$ & 0.0022 \\
\hline $\mathrm{C}_{2} \mathrm{WC} \mathrm{C}^{\prime} \mathrm{F} 6 \quad \ldots \ldots$ & 0.0121 & $\mathrm{C}_{1} \mathrm{EWL}^{\prime} \mathrm{F} 6 \mathrm{~F} 15 \quad \ldots$ & 0.0021 \\
\hline $\mathrm{ER}_{2} \mathrm{X}_{2} \mathrm{C}^{\prime \prime} \mathrm{F} 1,6$ & 0.0108 & $\mathrm{C}_{1} \mathrm{EX}_{\downarrow} \mathrm{L}^{\prime} \mathrm{F} 6 \ldots$ & 0.0021 \\
\hline $\mathrm{C}_{2}$ EWF6 $\ldots \ldots \ldots \ldots$ & 0.0095 & & \\
\hline
\end{tabular}

$$
\text { F. - S system }
$$

The analyses were performed in a way similar to that used for the two previous systems, considering, however, the non-linear subtypes in this system (Grosclaude \& Millot, 1962 ; Grosclaude, 1963, 1964) (table 7).

The notation $\mathrm{U}_{1}^{\prime}$ indicates the presence of $\mathrm{U}^{\prime}$ without $\mathrm{U}_{2}^{\prime}$. This phenogroup is characteristic of the Zebu breed (RIBAS, 1978 unpublished data) while it is not present in European breeds. 


\section{TABLE 7}

Frequency of the $S$ system phenogroups in the Cuban charolais

Fréquence des phénogroupes du système $S$ dans la race Charolaise cubaine

\begin{tabular}{|c|c|}
\hline Phenogroup & Frequency \\
\hline $\mathrm{UH}^{\prime} \mathbf{H}^{\prime \prime} \mathrm{U}^{\prime \prime} \ldots \ldots \ldots \ldots \ldots \ldots \ldots \ldots \ldots \ldots \ldots$ & 0.4140 \\
\hline $\mathbf{H}^{\prime} \ldots \ldots \ldots \ldots \ldots \ldots \ldots \ldots \ldots \ldots \ldots \ldots \ldots \ldots \ldots$ & 0.2376 \\
\hline 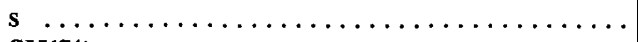 & 0.1194 \\
\hline 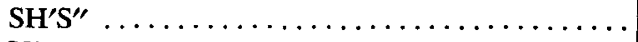 & 0.1047 \\
\hline $\mathrm{U}^{\prime} \ldots \ldots \ldots \ldots \ldots \ldots \ldots \ldots \ldots \ldots \ldots \ldots \ldots \ldots \ldots$ & 0.0370 \\
\hline $\mathbf{U H}^{\prime} \mathbf{H}^{\prime \prime} \ldots \ldots \ldots \ldots \ldots \ldots \ldots \ldots \ldots \ldots \ldots \ldots \ldots \ldots$ & 0.0383 \\
\hline $\mathbf{H}^{\prime} \mathbf{S}^{\prime \prime} \ldots \ldots \ldots \ldots \ldots \ldots \ldots \ldots \ldots \ldots \ldots \ldots \ldots \ldots \ldots$ & 0.0239 \\
\hline 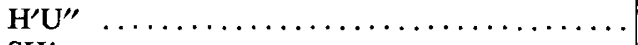 & 0.0075 \\
\hline $\mathbf{S H}^{\prime} \ldots \ldots \ldots \ldots \ldots \ldots \ldots \ldots \ldots \ldots \ldots \ldots \ldots \ldots \ldots$ & 0.0071 \\
\hline 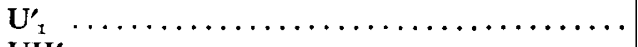 & 0.0044 \\
\hline $\mathbf{U H}^{\prime} \quad \ldots \ldots \ldots \ldots \ldots \ldots \ldots \ldots \ldots \ldots \ldots \ldots \ldots \ldots \ldots$ & 0.0038 \\
\hline $\mathbf{U}_{:}^{\prime}, \ldots \ldots \ldots \ldots \ldots \ldots \ldots \ldots \ldots \ldots \ldots \ldots \ldots \ldots \ldots$ & 0.0023 \\
\hline
\end{tabular}

It may therefore be concluded that the Cuban Charolais showed a great variability. Furthermore, our data showed that the Cuban Charolais differs from other breeds found in Cuba. However, the presence of the allele $U_{1}^{\prime}\left(U^{\prime}\right.$ without $\left.U_{2}^{\prime}\right)$ may indicate that Bos indicus «blood» is present in Cuban Charolais.

Reçu pour publication en juillet 1981.

\section{Acknowledgements}

We would like to thank Dr F. Grosclaude and his co-workers (Laboratoire de Génétique biochimique, Département de Génétique, Centre de Recherches zootechniques, 78350 Jouy-en-Josas, France) for their contribution to this work. Thanks are also given to the workers of the "Manuel Fajardo» Genetic Centre and to the technicians of the Immunogenetics Laboratory of the University of Havana whose co-operation made this work possible.

\section{Résumé}

Fréquences alléliques dans les systèmes de groupes sanguins $d u$ Charolais cubain

Un total de 223 couples mère-fils de la race Charolais cubaine ont été utilisés pour établir les fréquences alléliques dans les 11 systèmes de groupes sanguins actuellement connus $\left(\mathrm{A}, \mathrm{B}, \mathrm{C}, \mathrm{F}, \mathrm{J}, \mathrm{L}, \mathrm{M}, \mathrm{S}, \mathrm{Z}, \mathrm{R}^{\prime}, \mathrm{T}^{\prime}\right)$. Cinquante-huit allèles ont été trouvés dans le système $\mathrm{B}$ et quarante-trois allèles dans le système $C$. Les dix allèles les plus fréquents dans le Charolais cubain ne sont pas retrouvés dans les races Holstein, Santa Gertrudis, Criollo (Créole) ou Zébu. Les résultats de ce travail indiquent que le Charolais cubain recèle une grande variabilité génétique. Cette race est différente des autres races bovines à Cuba. Néanmoins, la présence de l'allèle $U^{\prime}{ }_{1}$ ( $U^{\prime}$ non $U^{\prime}{ }_{2}$, non observé dans les races européennes) indique l'existence dans le Charolais cubain de gènes provenant de Bos indicus. 